Databases, Revenues, \& Repertory: The French Stage Online, 16801793 • Données, recettes \& répertoire: La scène en ligne (1680-1793)

\title{
Introduction - Histoire du théâtre et humanités numériques
}

Sylvaine Guyot, Jeffrey S. Ravel

Published on: Oct 07, 2020

DOI: 10.21428/671d579e.e139b5e7

License: Creative Commons Attribution 4.0 International License (CC-BY 4.0). 
Au début du mois de juin 2018, l'équipe éditoriale de MIT Press nous fit part d'une nouvelle inattendue : l'ensemble de nos articles en ligne avait par mégarde été supprimé de la plateforme numérique et, de manière quelque peu surprenante, le fournisseur privé n'avait pas pris soin d'en faire une sauvegarde. La totalité de notre travail, avec celui de nos collaboratrices et collaborateurs, s'était donc évaporée. Nombre de courriels fiévreux et de réunions d'urgence s'ensuivirent ; de plates excuses nous furent adressées; des solutions de rechange furent proposées. Grâce à la réactivité de l'équipe de MIT Press, combinée à la prévoyance de nos contributrices et contributeurs qui avaient eu la sagesse de conserver une copie de leur article, nous pûmes recomposer le recueil et migrer sur une plateforme éditoriale plus stable, développée au Media Lab du MIT et adoptée par MIT Press. Une fois la catastrophe endiguée, nous reprîmes le travail et finîmes par mener à bien le présent volume ; mais c'est peu de dire que l'expérience fut déroutante. D'un jour à l'autre, des heures de travail menées sous l'égide de une presse universitaire de renom, elle-même associée au principal institut de technologie du monde, étaient parties en fumée. Si un tel accident pouvait se produire au MIT, quelle confiance accorder aux nouvelles technologies qui investissent actuellement le champ des sciences humaines et sociales ? Et alors que nous achevons aujourd'hui cette entreprise éditoriale, comment avoir la garantie que l'ouvrage que nous mettons en ligne existera encore dans un an, pour ne pas dire dans une ou dix décennies?

Si cette part d'incertitude autour de l'accès et de la préservation à long terme est familière aux chercheurs, archivistes et conservateurs chargés de la mise en ligne et de la sauvegarde de collections matérielles ou de sources nativement numériques, elle n'est pas non plus étrangère aux historiens du théâtre. En effet, leur objet d'étude - les spectacles vivants du passé - est lui-même un événement éphémère, immatériel, ou du moins insaisissable dans sa totalité, résistant donc fondamentalement à l'investigation historienne. Pour mener son enquête, l'historien du théâtre d’Ancien Régime ne dispose que de traces manuscrites ou imprimées du spectacle, ainsi que de quelques images immobiles, et ce, comme le rappelle Juliette Cherbuliez dans le présent volume, en l'absence de tout document sonore. L'archive considérée est donc par nature fragmentaire, lacunaire, irrémédiablement infidèle. Dès lors, avant de s'interroger sur la possible fragilité de l'accès que les nouvelles technologies donnent aux sources historiques, c'est aux lacunes des sources mêmes que l'historien est d'abord confronté. S'il est vrai que tous les champs de la discipline historienne se heurtent à de semblables trous, c'est particulièrement le cas pour l'histoire du théâtre, qui 
s'intéresse au moment de la séance, c'est-à-dire à la rencontre, en grande partie non documentée, entre les interprètes et leurs publics.

La mesure d'incertitude qui caractérise la pratique numérique n'échappe pas aux contributrices et contributeurs de ce volume, qui prend aussi bien pour objet un cas singulier dans l'histoire du théâtre - celui de la Comédie-Française entre 1680 et 1793 - qu'un projet de recherche actuel en humanités numériques, le Projet des Registres de la Comédie-Française ( $\underline{\mathrm{RCF}})$. Ce dernier, accessible gratuitement en ligne, est un programme transatlantique qui explore la façon dont les nouvelles technologies peuvent permettre d'étayer, d'enrichir, voire de modifier l'étude du passé théâtral. Le RCF est consacré à un ensemble unique et singulièrement complet d'archives manuscrites et imprimées, conservées à la Bibliothèque-Musée de la ComédieFrançaise, au Palais-Royal. Léquipe en charge du projet a procédé à la numérisation des 112 registres in-folio dans lesquels se trouvent consignées en détail les recettes quotidiennes liées à la vente des billets pour plus de 34000 représentations entre 1680 et 1793. Elle a ensuite extrait et transcrit ces données financières, qu'elle a rendues disponibles en ligne à travers trois outils de recherche et de visualisation, et qui sont accessibles dans leur totalité via une interface de programmation (API). Les premiers résultats de cette entreprise collective ont été publiés sur le site en 2014, avant de donner lieu, en 2015 et 2016, à une série de rencontres en France et aux États-Unis. Parmi elles, on mentionnera deux colloques internationaux,

Métamorphoses du corpus des registres de la Comédie-Française 1680-1793/2013-2016 (Paris, décembre 2015) et Pratiques théâtrales et Archives numérisées. Le Projet des Registres de la Comédie-Française (1680-1793) (Cambridge, Massachusetts, mai 2016) ; une journée d'étude consacrée aux approches économiques des registres (MIT, septembre 2016); et une série d'ateliers franco-américains au niveau master et doctorat organisés à l’Université Paris Nanterre et à l’Université Harvard (sous l'égide du programme IDEFI-CréaTIC et du Partner University Fund de la FACE Foundation). Ces rencontres associèrent la présentation de recherches menées à partir de la base RCF et des séances de travail collectif où informaticiens, jeunes chercheurs et universitaires combinèrent leurs efforts pour concevoir ensemble de nouvelles façons d'interroger les données et de nouveaux outils d'exploration. À ces occasions, l'on vit aussi des étudiants et des professionnels interpréter des pièces ou des extraits de pièces du répertoire de la Comédie-Française qui pour certaines n'avaient pas été jouées depuis leur création. Le mode d'investigation, de collaboration transdisciplinaire et de création artistique auquel ces événements donnèrent lieu a depuis encouragé leurs participants à renouveler leur approche de l'histoire du 
théâtre, et à imaginer des manières inédites de façonner pédagogie et pratique scénique à partir de ce corpus d'archives numérisées et de ces instruments de recherche.

Certaines de ces réalisations sont consultables sur le site du Programme $\underline{\mathrm{RCF}}$; les membres du projet, organisatrices et organisateurs de ces colloques, ont par ailleurs coordonné plusieurs publications collectives. Le Sacre de l'acteur (2017), qui rassemble les actes du colloque RCF qui s'est tenu en 2014 à l’Université de Poitiers, analyse l'essor des discours sur la célébrité dans le champ des arts du spectacle de 1650 à 1850ํㅗ. Un second recueil, publié dans Littératures classiques en 2018, et qui réunit onze des interventions présentées lors des colloques RCF, examine d'un point de vue historique la constitution et l'institutionnalisation du répertoire aux $\mathrm{XVII}^{\mathrm{e}}$ et XVIII ${ }^{\mathrm{e}}$ siècles $\underline{2}$, tandis qu'un numéro de la revue Théâtre/Public comprend une série d'entretiens avec des artistes de langue anglaise et française qui envisagent la pratique de ce répertoire sur la scène contemporaine ${ }^{3}$. Le présent volume s'inscrit assurément dans la continuité de ces publications antérieures : les réflexions proposées ici ont elles aussi été initialement exposées à l'occasion de rencontres RCF, soit lors du colloque de Paris en 2015 (Lauren R. Clay et Pierre Frantz), soit lors du colloque de Cambridge en 2016 (Jeffrey S. Ravel, Jeffrey Peters, Juliette Cherbuliez et William Weber), soit lors de la journée d'étude au MIT en 2016 (François Velde et Thomas M. Luckett) ; elles ont donc été élaborées de conserve avec les analyses qui figurent dans le numéro de Littératures classiques paru en 2018 et font également écho à la question du répertoire à laquelle s'attache le numéro de Théâtre/Public de 2017.

Mais cet ouvrage ne se distingue pas moins de manière significative des publications précédentes du RCF. Sa nouveauté réside, de toute évidence, dans son usage et son approche des ressources numériques. En premier lieu, si nous avons opté d'emblée pour une publication en ligne, c'est pour qu'elle soit accessible gratuitement, conformément à la politique de libre accès qui est au cœur même du Projet $\underline{\mathrm{RCF}}$; nous sommes heureux que MIT Press ait accepté de soutenir cette décision. En outre, nous ne souhaitions pas de version imprimée, car nous voulions inciter notre lectorat à considérer les possibles continuités entre l'interrogation de la base de données (sur le site) et la consultation (en ligne) des résultats de cette enquête. À cet égard, la publication numérique nous a semblé offrir un support plus favorable à la visualisation des données : nombreux sont ici les essais à inclure des graphiques qui sont plus attrayants et plus aisément lisibles qu'ils ne le seraient sur une page imprimée. En 
outre, dans certains cas, nous avons intégré dans le texte les outils de recherche/visualisation et les images numérisées des registres disponibles sur le site $\underline{\mathrm{RCF}}$; cette option permet au lecteur de vérifier lui-même les interprétations proposées, voire d'explorer d'autres questions que la lecture de l'article est susceptible de faire surgir. Enfin, les essais de la Section I interrogent explicitement les apports des humanités numériques à la recherche, question qui traverse également les essais et les commentaires qui suivent ; notre objectif est ainsi que les lecteurs de cet ouvrage en ligne réfléchissent conjointement à l'histoire culturelle et théâtrale du $\mathrm{XVIII}^{\mathrm{e}}$ siècle et aux nouvelles voies ouvertes dans les sciences humaines par les nouvelles technologies.

Le volume est divisé en trois sections. La première, intitulée « Interpréter les archives : données, visualisations, sons », examine la portée heuristique et les enjeux épistémologiques des approches numériques en histoire du théâtre. Dans « La Comédie-Française par les chiffres, 1752-2020», Jeffrey S. Ravel analyse trois études anciennes consacrées aux informations administratives contenues dans les registres de la Comédie-Française, en montrant que chacune a pris forme selon les conditions socioculturelles du contexte historique où elles ont été écrites. D’un point de vue historiographique, sinon généalogique, ces études signalent que la recherche quantitative existait bien avant l'émergence des technologies dont nous disposons aujourd'hui et, de ce fait, elles permettent de mieux saisir et de mieux mesurer les apports, les possibilités et les limites de l'outil numérique. Jeffrey Peters, pour sa part, observe la manière dont la visualisation de données, loin d'être le médium neutre d'un savoir figé, est une forme opérante qui reconfigure et renouvelle les questionnements traditionnellement formulés par la critique et l'histoire littéraires. Comparant les visualisations du Projet RCF à celles développées par Franco Moretti et d'autres chercheurs, «Voir le littéraire : la visualisation de données et le Projet des Registres de la Comédie-Française » nous invite à réfléchir aux présupposés méthodologiques, aux procédures d'enquête et à la nature des résultats propres au traitement quantitatif des corpus littéraires. Quant à Juliette Cherbuliez, c'est en regardant les images numérisées des registres quotidiens de recettes de la Comédie-Française sur son écran qu'elle s'est trouvée conduite à s'interroger sur le spectre des sensations éprouvées par les spectateurs dans la salle, lesquelles ne sont pas documentées dans les archives. Né du constat perceptif de cette absence, son article, «Les sons du théâtre : publics,

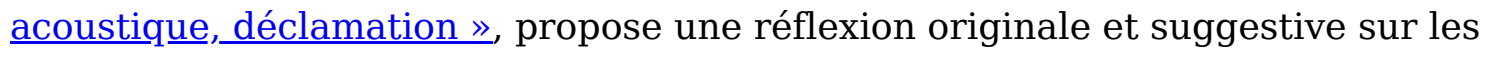
paysages sonores du passé et sur les nombreux projets d’humanités numériques qui entreprennent de recréer virtuellement, pour les analyser, ces expériences auditives. 
En considérant les objections formulées à leur encontre aussi bien qu'en faisant valoir les renouvellements qu'elles permettent, les trois essais de cette première section témoignent du fait que les sciences humaines ont beaucoup à gagner dans un usage éclairé des méthodologies numériques : de nouvelles façons d'approcher, de voir, d'écouter le passé. Ils n'en soulignent pas moins les zones silencieuses des ressources computationnelles, qui à elles seules ne suffisent jamais à saisir dans leur entièreté ni l'esthétique des productions artistiques ni la politique de l'institution théâtrale. Comme l'architecture tabulaire des registres du XVIII ${ }^{\mathrm{e}}$ siècle, les outils technocognitifs des humanités numériques ne sont pas de simples mécanismes d'enregistrement ou de restitution exhaustifs : ils circonscrivent le phénomène culturel dont ils rendent compte à une taxonomie de traits pertinents - ils le cadrent, et le cartographient -, déterminant autant qu'ils la nourrissent la représentation que l'on en a, et que l'on en donne.

La deuxième section, « Répertoire et recettes au XVIII' siècle », se compose de deux articles consacrés aux choix de programmation et aux stratégies commerciales de la Comédie-Française sur la période longue couverte par la base de données du Projet RCF. Spécialiste des scènes musicales et théâtrales dans l'Angleterre et l'Europe des $\mathrm{XVIII}^{\mathrm{e}}$ et XIX ${ }^{\mathrm{e}}$ siècles, William Weber, dans « Les répertoires parallèles de l’Opéra et de la Comédie-Française à la fin de l'Ancien Régime », compare la proportion de nouveautés et de pièces anciennes dans les répertoires respectifs de la ComédieFrançaise et de l'Académie royale de Musique. Il montre ainsi que les deux institutions royales traversent, dans les dernières décennies de l'Ancien Régime, une « crise » notable, liée aux évolutions de l'opinion publique qui tend à réclamer une présence plus régulière de nouveautés à l'affiche. D’un côté, ces débats sur le canon conduisent l'Opéra à s'ouvrir aux courants opératiques européens et à établir ainsi un répertoire qui s'avère suffisamment stable pour résister aux soubresauts révolutionnaires ; inversement, l'engouement pour les auteurs et les genres nouveaux plonge la ComédieFrançaise dans une crise qui aboutira dans les années 1790 à la fermeture du théâtre. Dans son ample contribution «Une analyse des recettes de la Comédie-Française, 1680-1793 $»$, François Velde, historien de l'économie, aborde les registres des recettes de la Comédie-Française comme un ensemble inégalé de sources sur la gestion d’une entreprise au XVIII ${ }^{\mathrm{e}}$ siècle. Considérant les revenus du théâtre sur le long terme, il étudie le lien que la fluctuation de ces derniers entretient avec la politique monétaire royale, comme avec les stratégies de prix et les décisions de programmation de la troupe. Le résultat majeur de cette étude est de montrer que, même ajustés en fonction de l'inflation, les revenus ne cessèrent d'augmenter après 1748 . Toute étude 
sur l'évolution des genres dramatiques et du répertoire à la Comédie-Française avant la Révolution se devra désormais de prendre en considération cette démonstration de la réussite commerciale de la compagnie.

Mis en série, ces deux articles offrent un éclairage remarquable sur les modalités et les déterminations de la programmation et de la politique tarifaire adoptées par les Comédiens Français entre l'âge du théâtre « classique » sous Louis XIV et l'émergence du drame romantique après la Révolution. Si aucune de ces études n’aurait été possible sans les outils informatiques du Projet RCF, toutes deux révèlent combien la pratique culturelle considérée relève d'une multifactorialité qu'il convient de prendre en compte. Non seulement les données statistiques ne font ni preuve ni document en soi, mais surtout, elles ne prennent sens qu'à condition de les rapporter au feuilletage contextuel dont elles procèdent, sans qu'il soit toujours possible d'épuiser ni de hiérarchiser ces multiples contextes. Cette section fait ainsi apparaître que les approches numériques, moins que d'être immédiatement informatives, ont pour vertu première de complexifier l'étude du fait culturel, en tant qu'elles appellent - et requièrent - une analyse multidimensionnelle, consciente des liaisons qu'elle établit entre telle ou telle variable et de l'importance préférentielle qu'elle accorde à tel ou tel facteur.

La troisième section, «Autour de 1760 », se déplace de la macroanalyse - celle du répertoire et des stratégies commerciales sur un peu plus d'un siècle - à l'échelle de la microanalyse, en se concentrant sur les années clés qui suivirent le milieu du siècle. Dans sa contribution, «Troubles financiers et stratégies commerciales à la ComédieFrançaise pendant la guerre de Sept Ans », Thomas M. Luckett compare les dépenses quotidiennes et les revenus de la Comédie-Française à la fin des années 1750 et au début des années 1760 pour montrer que les bénéfices connaissent alors, aussi bien pour la troupe dans son ensemble que pour les comédiennes et comédiens individuellement, un déclin à court terme. Cette période, qui coïncide avec le conflit militaire européen de la Guerre de Sept Ans, voit la Comédie-Française contrainte de développer de nouvelles stratégies de programmation et de demander au pouvoir royal une réforme administrative qui lui permette d'éviter la banqueroute. Lanalyse de T. Luckett qui se concentre sur un empan chronologique court, limité à une décennie, offre un contrepoint intéressant au constat fait par F. Velde d'une augmentation constante des revenus sur le long terme. Pierre Frantz identifie également un tournant dans les années 1760. En examinant précisément les registres de cette période, il montre qu'une série de choix de la Comédie-Française témoigne de ce qu’il appelle « le Moment Voltaire ». Voltaire, dont la première pièce fut créée en 1718 , 
tient dès 1760 une place prédominante dans la programmation de la troupe ; à cette date, les seize pièces du philosophe jouées plus de cent fois au XVIII ${ }^{\mathrm{e}}$ siècle sont déjà entrées au répertoire. Tandis que le trio des auteurs «classiques »-Molière, Racine et Pierre Corneille - était devenu le fonds patrimonial de la troupe, P. Frantz estime que le succès des pièces de Voltaire tient à la renommée littéraire et à la notoriété politique de l'auteur. La Comédie-Française bénéficia financièrement des multiples causes célèbres dont Voltaire prit la défense au cours des deux dernières décennies de sa vie. Dans la dernière contribution de cette section, «L'étrange carrière de Voltaire, le dramaturge le plus rentable du XVIII siècle », Lauren R. Clay donne à son tour la mesure du rôle majeur joué par le théâtre voltairien dans le répertoire et la stabilité économique de la Comédie-Française du vivant du dramaturge. À l'instar de F. Velde et T. Luckett, elle prend en considération, outre la fréquence avec laquelle ses pièces sont mises à l'affiche, les recettes qu'elles rapportent à la troupe. Elle montre en particulier que, dans les années 1760, l'œuvre de Voltaire était devenue beaucoup plus rentable pour l'institution parisienne que celle de l'autre auteur alors le plus joué du répertoire, Molière. Confirmant la prédominance mise en lumière par P. Frantz après 1750, L. Clay se tourne in fine vers le siècle suivant pour tenter de comprendre pourquoi les pièces de Voltaire disparurent complètement du répertoire au milieu du XIX $\mathrm{X}^{\mathrm{e}}$ siècle et pourquoi l'histoire littéraire aujourd'hui ne voit que rarement en Voltaire un grand auteur dramatique, ignorant l'importance qu'il eut sur la scène du XVIII ${ }^{\mathrm{e}}$ siècle.

Ainsi, ces trois derniers essais situent avec fécondité une analyse des facteurs internes qui motivent les choix de programmation de la Comédie-Française dans le contexte de certains événements qui adviennent hors du théâtre au cours de la génération précédant la Révolution. Attentive aux régularités des grandes tendances comme aux discontinuités de certains moments singuliers, cette section témoigne des jeux d'échelles possibles au sein de l'enquête, qu'il s'agisse des différentes échelles de temps, des échelles d'action ou des échelles de la réalité socio-culturelle à l'aune desquelles les données peuvent être interprétées.

La publication de ce volume vient conclure un premier cycle d'analyses entamé lors de la mise en ligne inaugurale du Projet $\underline{\mathrm{RCF}}$ en 2014. Il ne met cependant pas fin à nos investigations sur les archives numériques que nous avons constituées. La phase suivante, déjà en cours, s'attache à extraire et exploiter les données contenues dans les registres qui consignent les dépenses quotidiennes de la troupe, pour pouvoir les considérer en regard des données sur les revenus. Cette nouvelle série d'informations permettra aux chercheurs d'approfondir et de préciser l'étude des profits de l'institution sur des périodes plus ou moins longues du XVIII ${ }^{\mathrm{e}}$ siècle. (Ce travail 
bénéficie du soutien de l'Agence nationale de la recherche.) Parallèlement, nous traitons actuellement les données contenues dans les registres des feux, qui indiquent la distribution pour chaque représentation. Cet autre ensemble devrait permettre aux historiens d'évaluer plus précisément l'impact de la popularité des interprètes sur les revenus et les décisions de programmation. (L'initiative est financée par le Conseil de recherches en sciences humaines du Canada.) Enfin, une nouvelle équipe de spécialistes du théâtre du XIX ${ }^{\mathrm{e}}$ siècle a commencé à travailler avec Agathe Sanjuan, conservatrice de la Bibliothèque-Musée de la Comédie-Française, pour numériser les documents administratifs de la troupe depuis sa recomposition en 1799 jusqu'au début de la Première Guerre mondiale. Une fois ces données ajoutées, le RCF offrira un aperçu sans égal du fonctionnement d'une des principales institutions culturelles du monde occidental pendant plus de deux siècles.

Si l'avenir du projet est donc en un sens prometteur, demeure néanmoins toujours l'ombre des incertitudes évoquées au seuil de cette introduction. Lorsque le projet prit forme, nous pensions que les images numérisées des registres, les bases de données et les outils de recherche et de visualisation trouveraient un hébergement durable sur les serveurs du MIT. Mais l'infrastructure de stockage des données au MIT fut ces dernières années soumis à des changements constants, ce qui nous conduisit à transférer le projet sur des serveurs commerciaux. Nos partenaires français, en particulier la Comédie-Française, exprimèrent alors une certaine inquiétude à l'idée de voir le patrimoine numérisé français confié aux intérêts privés d'entreprises américaines. Aussi avons-nous, à la fin 2018, initié le transfert de toutes nos données vers Calcul Canada, un service d’hébergement gratuit géré par le gouvernement canadien pour soutenir la recherche dans les universités publiques du pays. Une fois cette migration achevée, nous créerons un site miroir sur Huma-Num, une plateforme de serveurs française, également mise en œuvre par l'État et destinée à stocker les données numériques des chercheurs en sciences humaines et sociales. Ceux d'entre nous qui, dans l'équipe RCF, occupent des postes dans des universités américaines ne peuvent qu'admirer l'accessibilité et la longévité promises par ces infrastructures publiques.

Nous souhaiterions pour conclure nous adresser, non sans un certain optimisme, à nos lecteurs de l'an 2150 : si vous achevez la lecture de cette introduction, nous sommes heureux que ce dialogue ait pu se nouer de nous à vous. Que vous ayez accès à ce texte signifie que la communauté scientifique est parvenue à développer, pour assurer la préservation des archives numériques du XXI ${ }^{\mathrm{e}}$ - et du XVIII ${ }^{\mathrm{e}}$ - siècles, une technologie aussi durable que le codex. 


\section{Footnotes}

1. Florence Filippi, Sara Harvey et Sophie Marchand (dir.), Le Sacre de l'acteur. Émergence du vedettariat théâtral de Molière à Sarah Bernhardt, Malakoff, Armand Colin, 2017.

2. Christian Biet (dir.), La Question du répertoire au théâtre, Littératures classiques, $\mathrm{n}^{\circ}$ 95, 2018.

3. Christian Biet (dir.), Le Répertoire aujourd'hui, Théâtre/Public, $\mathrm{n}^{\circ} 225$, juilletseptembre 2017. $\_$ 